\title{
Patterns of Traditional and Nontraditional Network Involvement in Insulo-Sylvian Gliomas: An Anatomic Study using the Quicktome Platform
}

\section{Zhiqiang Wu}

The First Affiliated Hospital of Nanjing Medical University

\section{Zifeng Zhang}

The First Affiliated Hospital of Nanjing Medical University

Guanjie Hu

The First Affiliated Hospital of Nanjing Medical University

\section{Xiefeng Wang}

The First Affiliated Hospital of Nanjing Medical University

\section{Lin Zhao}

The First Affiliated Hospital of Nanjing Medical University

\section{Binbin Wang}

The First Affiliated Hospital of Nanjing Medical University

Ning Liu

The First Affiliated Hospital of Nanjing Medical University

Nicholas Dadario

Rutgers University

\section{Yan Zheng}

Omniscient Neurotechnology

Junxia Zhang

The First Affiliated Hospital of Nanjing Medical University

Michael Sughrue

Omniscient Neurotechnology

Yongping You (D YYPL9@njmu.edu.cn )

The First Affiliated Hospital of Nanjing Medical University

\section{Research Article}

Keywords: Network, Machine learning, Gliomas, Insula, Sylvian, Language, Cognition

Posted Date: January 17th, 2022

DOI: https://doi.org/10.21203/rs.3.rs-1222638/v1 
License: (c) (i) This work is licensed under a Creative Commons Attribution 4.0 International License. Read Full License 


\section{Abstract}

\section{Background}

Neurosurgeons are increasingly capable of maintaining language and motor functions in glioma patients following surgery due to the ability to preserve traditionally "eloquent" structures. However, glioma patients continue to present with severe morbidity in cognitive functions due to the lack of familiarity in the neurosurgical community with non-traditionally "eloquent" brain networks. Therefore, the authors sought to identify and describe the frequency of invasion and/or proximity of Insulo-Sylvian gliomas to portions of non-traditional, large scale brain networks during surgery.

\section{Methods}

A retrospective analysis was completed of consecutive adult patients undergoing surgery for newly diagnosed glioma at a single center between 2017-2020 with WHO grade II-IV infiltrating gliomas centered in the insula, opercular cortices or temporal stem. Diffusion tensor imaging (DTI)-based tractography was completed by creating a personalized brain connectome atlas based on the Human Connectome parcellation scheme with Quicktome software. This algorithm utilizes an machine learning $(\mathrm{ML})$-approach to assign voxels of the cerebral cortex to various brain regions according to structural connectivity patterns of voxels in the brain region of interest utilizing neuroimaging data specifically from normal healthy adults. Insulo-Sylvian tumors were categorized based on their involvement with nontraditional cognitive networks versus traditionally eloquent structures.

\section{Results}

45 patients were included ( $47 \pm 15$ years, $51 \%$ female) consisting of mostly high grade (IV)-gliomas (56\%) compared WHO grade II (22\%) or III (22\% tumors). Ultimately, 44/45 (98\%) patients demonstrated tumor involvement ( $<1 \mathrm{~cm}$ proximity or invasion) to the cortical or subcortical components of a non-traditional, large scale brain network or major white matter pathway involved in cognition. In comparison, 35/45 (78\%) patients demonstrated tumor involvement of traditionally considered "eloquent" structures like the corticospinal tract or language regions/tracts. The most common non-traditional cognitive networks involved in cases included the salience network (60\%) followed by the central executive network (56\%).

\section{Conclusions}

Non-traditionally "eloquent" brain networks are increasingly encountered during surgical resection of Insulo-Sylvian gliomas in both hemispheres and must be considered moving forward. Damage or dysfunction in these networks has been shown to result in severe cognitive morbidity and an improved understanding of their presence can allow for more informed surgical decisions based on patient oncofunctional goals.

\section{Introduction}


It is well established that performing intra-axial tumor surgery around the Sylvian fissure, insula, and opercular cortices has a significant risk of causing new and permanent neurological deficits (1). A substantial body of literature has been dedicated to refining techniques for reducing this risk. Most work has principally focused on reducing risks of causing motor weakness, and aphasia with left sided lesions, where the anatomy has been known for some time. However, more recent efforts have focused on functional preservation techniques in less well-known anatomy, such as preservation of the inferior frontal-occipital fasciculus (IFOF) (2) and the frontal aslant tract (FAT) $(3,4)$. While it is generally wise to avoid cutting through the middle of white matter bundles, removing an intra-axial brain tumor always requires cutting some brain tissue, reflecting our constant need to make trade-offs in surgical choices. However, it remains clear that numerous glioma patients continue to present with severe post-operative morbidity in higher order functions (5-7), limiting their return back into society and the workplace(8). Given that neurological decline occurs after brain surgery in many cases (9), especially despite the fact that no serious transgressions of white matter bundles occurred, it is clear that additional information is necessary to define the anatomy at risk in these operations.

Recent work in neuroscience has clearly established the central role of large scale brain networks, such as the Central Executive Network (CEN) (10-13), Default Mode Network (DMN) (14-17), Salience network (SN) (12, 18-20), Dorsal Attention network (DAN) $(10,21,22)$, and Ventral Attention network (VAN) (22), in numerous aspects of human cognition. These networks include spatially distant, but highly synchronized brain regions and their connecting white matter fibers which dynamically work together across the human cortex. While specific aspects of these networks when damaged by brain tumors or dysfunctional in neuropsychiatric illnesses have yet to be completely mapped to specific clinical symptoms, it is increasingly clear that inadvertently injuring these networks during surgery is a mechanism by which patients experience increased cognitive morbidity following surgery(23). To date, the large-scale brain networks have provided the best anatomic maps to make sense of traditionally less familiar anatomy responsible for cognitive functions. Therefore, the large-scale brain networks during glioma surgery is a topic which demands increased scrutiny moving forward.

For some time, progress in answering these kinds of questions has been held back by the substantial challenge of meaningfully mapping structural connections and making sense of them in cases where gliomas have distorted and/or invaded these connections. However, the Quicktome algorithm addresses these limitations by utilizing a machine learning (ML)-approach which assigns voxels of the cerebral cortex to various regions based on their similarity of structural connectivity patterns of voxels of this region seen in normal healthy adults. Therefore, in this study, we utilized Quicktome to characterize a cohort of patients with gliomas around the insula and sylvian fissure as it relates to traditional and nontraditional brain networks. While we expect that these cases will demonstrate that often these tumors are in areas well known to neurosurgeons to be "eloquent" structures, like the language system and sensorimotor cortices, our specific goal was to demonstrate the frequency of invasion and/or proximity to portions of non-traditional, large scale brain networks such as the CEN, DMN, SN, VAN and DAN. 


\section{Methods}

\section{Patient Cohort}

The patients in this study were retrospectively analyzed from a consecutive series of adult patients undergoing surgery for newly diagnosed glioma at our center between 2017 and 2020 with WHO grade II to IV infiltrating gliomas centered in the insula, opercular cortices or temporal stem. While many of these tumors extend beyond these regions, we included only patients where the primary epicenter of the tumor was in the insulo-sylvian regions. This project was completed with Human Ethics Committee approval of the first affiliated hospital of Nanjing Medical University, and all patients provided informed consent to study participation and image publication.

\section{Imaging protocol}

All patients underwent a pretreatment standard structural T1 and T2 weighted images used for image guidance immediately prior to surgery. In addition, they underwent diffusion tractography imaging (DTI) with the following parameters: Siemens Skyra 3.0 MRI scanner, with $10 \mathrm{~b}=0$ baseline image and a $b=1000$ shell with 64 direction acquisition, $F O V=224 \mathrm{~mm} \star 224 \mathrm{~mm}$, slice thickness $2 \mathrm{~mm}, 0 \mathrm{~mm}$ gap between slices with no overlap, full brain coverage, isotropic voxels, square $112 * 112$ matrix.

\section{Diffusion tractography preprocessing steps}

The DT images were processed using the Omniscient Quicktome software (24), which employs a standard processing steps in the Python language (25) which specifically include the following steps: 1) the diffusion image is resliced to ensure isotropic voxels, 2) motion correction is performed using a rigid body alignment, 2) slices with excess movement (defined as DVARS $>2$ sigma from the mean slice) are eliminated, 3) the T1 image is skull stripped using a convolutional neural net (CNN), this is inverted and aligned to the DT image using a rigid alignment, which is then used as a mask to skull strip the DT, 4) gradient distortion correction is performed using a diffeomorphic warping method which aims to locally similarize the DT and T1 images, 5) eddy current correction is performed, 6) fiber response function is estimated and the diffusion tensors are calculated using constrained spherical deconvolution, 7) deterministic tractography is performed with random seeding, usually creating about 300,000 streamlines per brain.

Creation of a personalized brain atlas using machine learning based parcellation

The Quicktome algorithm creates a ML-based, subject specific version of the HCP-MMP1 (26) atlas based on diffusion tractography structural connectivity, which has been reported in previous studies (27). In short, this was created by training a ML-model on 200 normal adult subjects by first processing T1 and DT images as above. A HCP-MMP1 atlas in NIFTI MNI space is then warped onto each brain to assess structural connectivity between each parcellation pair of the atlas and a set of regions including 8 subcortical structures per hemisphere and the brainstem based on streamlines terminating in each region. This step both allows the generation of feature vectors (basically a $379 \times 379$ structural 
connectivity-based adjacency matrix), and generates a centroid of the parcellation which is utilized to constrain the voxels studied for assignment to a given parcellation to a plausible area in the vicinity of its typical position. These feature vectors for each region were then used as a training set and the data were modeled using the XGBoost method.

This model is then applied to the new subject by first warping the HCP-MMP1 atlas to the new brain and collecting a set of feature vectors of the connectivity of each voxel. The feature vectors are then used to determine if each voxel belongs to a parcellation or region or not, and if so to assign the voxel to that parcellation. This creates a version of the HCP-MMP1 atlas with subcortical components, which is not dependent on brain shape or pathologic distortion, and which is specific for this subject, but comparable between subjects.

\section{Definition of tumor boundaries for purposes of this analysis}

Tumor boundaries were defined using the preoperative postcontrast T1 and T2 images. For the purposes of this study, we used definitions of tumor boundaries to align with typical surgical goals as opposed to strict definition of all T2 changes in high grade gliomas being defined as tumor. Thus, the boundaries of enhancing high grade gliomas were defined solely as the contrast enhancing portion of the tumor, and the boundaries of low-grade gliomas were defined as the T2 hyperintense portion of the tumor. Of note, none of these patients received bevacizumab or other therapies which could change the imaging behavior in a clinically important way.

The rationale for limiting analysis of high-grade tumors to solely the contrast enhancing portions is two fold: First, the T2 changes in most Insulo-Sylvian high-grade gliomas are typically quite extensive, which would make this study biased towards overestimating the frequency of involvement of networks like CEN, DAN and SN in the surgical approach (they would be involved or near the T2 change in basically every case) and second, given the location of these tumors, it is unlikely that even advocates of supramaximal resectable surgery would recommend chasing $\mathrm{T} 2$ changes into the corticospinal tract, arcuate fasciculus and basal ganglia. Thus, we felt that given the goal of most neurosurgeons operating on a high-grade glioma centered in this region would be to remove as much contrast enhancing tumor as possible, we should perform our analysis in a way which aligned with the surgical goals of these cases to provide an accurate snapshot of the nature of nontraditional network involvement in a standard surgical neurooncology workflow.

\section{The determination of network involvement in glioma cases}

Quicktome defines networks as a set of cortical regions, defined using the machine learning based atlas described above, and the fiber bundles connecting the component regions. This structural connectivitybased approach to atlasing leverages the insight that specific cortical regions have been reproducibly and robustly demonstrated to have strong fMRI blood oxygen level dependent (BOLD) signal correlation with other regions in the brain, in patterns which are consistent across individuals. These correlation patterns form the basis of the concept of large-scale brain networks, for instance, regions of the brain which synchronize their activity because they serve a common function. The cortical regions used in Quicktome 
to select the anatomic components of these networks are determined from published meta-analyses which mapped the specific large scale brain networks to these specific regions $(28-33)$.

We visually inspected the processed images to determine whether the boundary of the tumor was in close proximity to (defined by tumor extending to within $1 \mathrm{~cm}$ of some portion of the tract or cortical region of a brain network template) or invaded various brain structures.

\section{Results}

\section{Patient cohort and characteristics}

A total of 45 patients met the inclusion criteria described above. The demographic data for these is outlined in Table 1. They had a median age at diagnosis of $47 \pm 15$ years, with a male to female ratio of 22 to 23 . There were 26 left sided tumors and 19 right sided tumors. There were $10 \mathrm{WHO}$ grade II gliomas, 10 WHO grade III gliomas, and $25 \mathrm{WHO}$ grade IV gliomas. While most of these tumors had some extension into the insula, and many were multilobular or large tumors, only 6/45 patients had a tumor largely focused in the insula, while 13 had tumors mostly focused in the frontal lobe and frontal opercula, 22 had primarily temporal tumors, and 4 tumors were mainly focused in the parietal opercula and inferior parietal lobule. 
Table 1

Subject demographics.

\begin{tabular}{|ll|}
\hline Variable ( $\boldsymbol{n}=45)$ & \\
\hline Age (mean, SD, in years) & $47(15)$ \\
\hline Gender & $22(49 \%)$ \\
\hline Female ( $n, \%)$ & \\
\hline Ipsilesional side & $26(58 \%)$ \\
\hline Left $(\mathrm{n}, \%)$ & \\
\hline WHO grade (n, \%) & $10(22 \%)$ \\
\hline Grade II gliomas & $10(22 \%)$ \\
\hline Grade III gliomas & $25(56)$ \\
\hline Grade IV gliomas & \\
\hline Location of the tumor $(n, \%)$ & $6(13)$ \\
\hline Insula & $13(29 \%)$ \\
\hline Frontal Predominant & $22(49 \%)$ \\
\hline Temporal Predominant & $4(9 \%)$ \\
\hline Parietal Predominant
\end{tabular}

Insulo-Sylvian gliomas usually involve both traditional and non-traditionally eloquent brain networks

We found that if we limited our analysis of whether an Insulo-Sylvian glioma was in close proximity to or invading a structure classically viewed as eloquent by neurosurgeons (specifically the corticospinal tract, the motor cortex, the language regions or tracts, the optic radiations, or the basal ganglia), around $77 \%$ of patients (35/45) demonstrated involvement of a traditional eloquent structure. Conversely 44/45 (98\%) patients demonstrated involvement (proximity or invasion) of a non-traditionally eloquent brain network, such as the CEN, SN, DMN, DAN or VAN. Ultimately, only 1 patient in this series did not demonstrate a surgically relevant risk to some structure, network or tract associated with a known neurological, or cognitive function.

An overall summary of the patterns of network involvement with these Insulo-Sylvian tumors is displayed in Table 2. Notably, the SN and its associated tract (FAT) were frequently involved, being nearby or invaded in $60 \%$ of these cases, as were the other more laterally positioned networks, CEN (56\% of cases) and VAN ( $63 \%$ of right sided cases as it is a right lateralized network). The more medial positioned DAN was affected slightly less often ( $47 \%$ of cases) and the DMN was relatively uncommon ( $18 \%$ of cases) 
with the involvement being most often due to proximity to the parietal component of the DMN with the medial frontal component invaded by tumor extension in one case.

Table 2

Overall summary of the patterns of network involvement with these Insulo-Sylvian tumors

\begin{tabular}{|lllll|}
\hline & & Uninvolved & $\begin{array}{l}\text { Closer Than } \\
1 \mathrm{~cm}\end{array}$ & $\begin{array}{c}\text { Structure } \\
\text { Invaded }\end{array}$ \\
\hline Cognitive networks & Salience & $18(40 \%)$ & $9(20 \%)$ & $18(40 \%)$ \\
\hline & CEN & $20(44 \%)$ & $8(18 \%)$ & $17(38 \%)$ \\
\hline & DMN & $37(82 \%)$ & $1(2 \%)$ & $7(16 \%)$ \\
\hline DAN & $24(53 \%)$ & $9(20 \%)$ & $12(27 \%)$ \\
\hline VAN & $32(71 \%)$ & $4(9 \%)$ & $9(20 \%)$ \\
\hline & Optic & $16(36 \%)$ & $22(49 \%)$ & $7(16 \%)$ \\
\hline & Radiations & & $7(16 \%)$ & $19(42 \%)$ \\
\hline Language & $19(42 \%)$ & $75(33 \%)$ \\
\hline Mhite Matter Tracts & Motor Cortex & $20(44 \%)$ & $10(22 \%)$ & $11(24 \%)$ \\
\hline CST & $10(22 \%)$ & $24(53 \%)$ & $6(13 \%)$ \\
\hline $\begin{array}{l}\text { Analysis Limited to Relevant } \\
\text { Hemisphere }\end{array}$ & Basal Ganglia & $19(42 \%)$ & $20(44 \%)$ & $15(33 \%)$ \\
\hline & FAT & $18(40 \%)$ & $12(27 \%)$ & $14(31 \%)$ \\
\hline & IFOF & $14(31 \%)$ & $17(38 \%)$ & $19(73 \%)$ \\
\hline & Language & $0(0 \%)$ & $7(27 \%)$ & $8(42 \%)$ \\
\hline
\end{tabular}

\section{Discussion}

In this study, we evaluated a cohort of patients who presented to our center with insular and peri-Sylvian gliomas and utilized a machine learning based analytic tool to determine the frequency of involvement of large-scale brain networks and/or associated en passage white matter fiber bundles in these tumors. We found that in these patients, greater than $98 \%$ of these patients had tumors which had sub-centimeter distance to the cortical or subcortical components of a large-scale brain network or major white matter pathway (or it invaded these structures). Most of these patients had multiple structures at risk from their tumor. All of this highlights the challenges posed by these complex tumors, and suggests that these cases really seldom are no risk, even when on in right hemisphere. 
There are substantial advantages to reconsidering the deep white matter anatomy in terms of large-scale brain networks. Notably, many large white matter bundles are in fact numerous bundles connecting different areas, not all of which are necessary for human cognitive function. This is especially true for the superior longitudinal fasciculus (SLF)-arcuate fasciculus (AF) complex, which is a highly complex network of connections, of varying importance. Given that some parts of these bundles and their branches are more important than others, and we need to cut something in order to remove a glioma, network-based approaches provide the potential benefit for better defining which white fibers are more important than others. Ultimately, all of brain surgery involves accurately calculating risks vs rewards of our actions.

\section{The Non-Traditional, Large Scale Brain Networks}

\section{Default Mode Network}

The default mode network (DMN) serves a primary role in passive states of mind (29), like internal thought or contemplation, however, it is also active during some goal-oriented tasks. The DMN network is typically described as consisting of the anterior and posterior cingulate cortices, and the lateral parietal lobe bilaterally. DMN also plays an integral role in coordinating with other networks for passive sensory processing (30). These connections often include: visual system (31), language subnetwork (32), and limbic system (33). In Figure 1, we demonstrate a tumor invading the DMN.

\section{Salience Network}

The Salience Network (SN) is integral for sensorimotor processing, general cognition, and coordinating between emotion, pain, and physical action (34). As the mind's moderator, the SN constantly monitors the external environment and decides how other brain networks react to new information and stimuli, it also plays an essential role in switching between the internal and external processing (35) of the brain's two main control networks (36): the default mode network (DMN) and central executive network (CEN). The main functional areas of the salience network are located in the anterior cingulate, the anterior insula (37, 38) and the presupplementary motor areas (39). The SN also includes nodes in the amygdala, hypothalamus, ventral striatum, thalamus, and specific brainstem nuclei (37), anterior cingulate cortex (ACC), medial temporal network, parahippocampal gyrus, olfactory lobe, and the ventral tegmental area (VTA). In Figure 2, we demonstrate a tumor invading the SN.

\section{Central Executive Network}

The central executive network (CEN) exists as a superordinate control network (40). It uses input from other networks for task selection and executive function. By integrating with the other brain networks, the CEN processes a varied set of information, such as flexibility, working memory, initiation, and inhibition, all of which had previously been thought to be separate processes. Since its initial discovery in the anterior frontal lobe (41),the central executive network has been found to be functionally connected to 
regions in the anterior cingulate cortex, the inferior parietal lobe (42), and the posterior most portions of the middle and inferior temporal gyri $(43,44)$. In Figure 3 , we demonstrate a tumor invading the CEN.

\section{Dorsal Attention Network}

The dorsal attention network (DAN) is an important mediator of goal-directed attentional processing (24), and it has many ways of contributing to intellectual capabilities. As a bilateral network, DAN demonstrates strong connectivity between areas in the lateral occipital lobe, the pre-central sulcus, the dorsal-most portion of the superior frontal sulcus considered to be the frontal eye fields (FEF), the ventral premotor cortex, superior parietal lobule, intraparietal sulcus, and motion-sensitive middle temporal area (45). In Figure 4, we demonstrate a tumor invading the DAN.

\section{Ventral Attention Network}

The ventral attention network (VAN) is one of two-network model of cortical attention $(46,47)$, which is involved in reorienting attention when a new, unexpected stimulus, like shock, frightening events, or "oddball" occurrences, is detected within the environment $(47,48)$. Multiple cortical areas, such as the middle and inferior frontal gyri, anterior insula, inferior parietal lobule, and temporo-parietal junction have been linked in this processing (25). In Figure 5, we demonstrate a tumor invading the VAN.

Limitations and Future Directions

The specific goal of this study was to estimate how often a surgeon performing surgery for infiltrating gliomas around the Sylvian fissure could reasonably expect that a network not normally addressed at surgery would be inside of the tumor, and this ultimately suggested that this was the expected nature of almost all of these tumors. This study is a survey of the insula-Sylvian region gliomas encountered in at a single center over a 3-year period. Ultimately, this study was not intended to be an exhaustive epidemiologic study of all patterns of glioma spread in cases around the Sylvian fissure, and it is possible that a different center may find different frequencies of network involvement with a similar cohort. We studied a consecutive series and generally aim to perform resectable surgery of some type for every case reasonable, even if not completely resected. Thus, there is not an obvious selection bias which skewed the results which is readily apparent to us other than the inherent referral patterns which route tumors around the Sylvian fissure frequently to university-based neuro-oncology programs. While it is possible that a cohort of gliomas seen over a large community cohort, with different complexity and disease severity from ours, might have different specific rates of involvement of the brain networks than our study, the fact remains that the rate of these networks being involved in these tumors is likely high. Therefore, they will continue to pose a risk of cognitive and emotional morbidity in the majority of patients if we continue to not address the situations where they cannot or should not be transgressed in the name of increasing the extent of resection a small amount.

\section{Conclusions}


In this study we demonstrate the extensive involvement of Insulo-Sylvian tumors with non-traditionally "eloquent" large-scale brain networks involved in cognition. While neurosurgeons have traditionally focused on eloquent structures related to language and motor activity, failure to consider the presence of non-traditional, large-scale brain networks will likely continue to exacerbate the cognitive and emotional disturbances documented in the majority of glioma patients after surgery. Brain network maps provide an improved understanding of the relationship between tumor and brain anatomy such that neurosurgeons can better understand the risks and benefits of certain surgical decisions for specific tumors.

\section{Declarations}

Ethics approval and consent to participate

The experimental protocol was established, according to the ethical guidelines of the Helsinki Declaration and was approved by the Human Ethics Committee of the first affiliated hospital of Nanjing Medical University. Written informed consent was obtained from individual or guardian participants.

\section{Consent for publication}

Not applicable.

\section{Availability of data and material}

All deidentified data used in the current manuscript can be provided by the authors per the readers requests. No data spreadsheet has been made publicly available due to patient confidentiality.

\section{Competing interests}

Michael Sughrue is the Chief Medical Officer, co-founder and share-holder of Omniscient Neurotechnology. Yan Zheng is an employee of Omniscient Neurotechnology. Products related to these companies were only discussed in this paper for discussion of methodology or statistical analyses. No other authors report any conflict of interest.

\section{Funding}

This study was supported by the grant from National Natural Science Foundation of China (81974389), Jiangsu Province's Key Discipline of Medicine (ZDXKA2016001), and the Priority Academic Program Development of Jiangsu Higher Education Institutions (PAPD).

\section{Author Contributions}

JZ, ZZ, GH, ZW, XW, LZ, BW, NL, and YY contributed to the conception and design of the study, data curation, and wrote sections of the manuscript. ND, YZ, MS, and YY did the statistical analyses and data interpretation and wrote the first draft of the article. All authors contributed to manuscript revision, read, and approved the submitted version. 


\section{Acknowledgements}

Not applicable.

\section{Authors' information}

${ }^{1}$ Department of Neurosurgery, The First Affiliated Hospital of Nanjing Medical University, Nanjing, 210029, China.

2 Institute for Brain Tumors, Jiangsu Collaborative Innovation Center for Cancer Personalized Medicine, Nanjing Medical University, Nanjing, 211166, China.

${ }^{3}$ Robert Wood Johnson Medical School, Rutgers University, New Brunswick, New Jersey, 08901

${ }^{4}$ Omniscient Neurotechnology, Sydney, NSW, 2000, Australia.

${ }^{5}$ Centre for Minimally Invasive, Neurosurgery, Prince of Wales Private, Hospital, Sydney, NSW, 2031, Australia.

${ }^{6}$ International Neuroscience Scientific Cooperation Center, XD Group Hospital, Xi'an, 710082, China.

\section{References}

1. J. Deverdun et al., Language reorganization after resection of low-grade gliomas: an fMRI task based connectivity study. Brain imaging and behavior 14, 1779-1791 (2020).

2. E. Caverzasi et al., Identifying preoperative language tracts and predicting postoperative functional recovery using HARDI q-ball fiber tractography in patients with gliomas. Journal of neurosurgery 125 , 33-45 (2016).

3. C. M. Baker et al., The crossed frontal aslant tract: A possible pathway involved in the recovery of supplementary motor area syndrome. Brain Behav 8, e00926 (2018).

4. B. L. Chernoff et al., A Role for the Frontal Aslant Tract in Speech Planning: A Neurosurgical Case Study. Journal of cognitive neuroscience 30, 752-769 (2018).

5. M. Dhandapani, S. Gupta, M. Mohanty, S. K. Gupta, S. Dhandapani, Trends in cognitive dysfunction following surgery for intracranial tumors. Surg Neurol Int 7, S190-S195 (2016).

6. C. Drewes, L. M. Sagberg, A. S. Jakola, O. Solheim, Perioperative and Postoperative Quality of Life in Patients with Glioma-A Longitudinal Cohort Study. World Neurosurgery 117, e465-e474 (2018).

7. L. M. Jenkins, K. J. Drummond, D. G. Andrewes, Emotional and personality changes following brain tumour resection. J Clin Neurosci 29, 128-132 (2016).

8. D. Starnoni et al., Returning to work after multimodal treatment in glioblastoma patients. Neurosurgical Focus FOC 44, E17 (2018). 
9. C. Niki et al., Primary Cognitive Factors Impaired after Glioma Surgery and Associated Brain Regions. Behavioural neurology 2020, 7941689 (2020).

10. P. Boord, T. M. Madhyastha, M. K. Askren, T. J. Grabowski, Executive attention networks show altered relationship with default mode network in PD. Neurolmage. Clinical 13, 1-8 (2017).

11. A. C. Chen et al., Causal interactions between fronto-parietal central executive and default-mode networks in humans. Proceedings of the National Academy of Sciences of the United States of America 110, 19944-19949 (2013).

12. K. K. Ellard et al., Functional Connectivity Between Anterior Insula and Key Nodes of Frontoparietal Executive Control and Salience Networks Distinguish Bipolar Depression From Unipolar Depression and Healthy Control Subjects. Biological psychiatry. Cognitive neuroscience and neuroimaging 3 , 473-484 (2018).

13. M. Firbank et al., Neural correlates of attention-executive dysfunction in lewy body dementia and Alzheimer's disease. Human brain mapping 37, 1254-1270 (2016).

14. T. Alderson et al., Disrupted Thalamus White Matter Anatomy and Posterior Default Mode Network Effective Connectivity in Amnestic Mild Cognitive Impairment. Frontiers in aging neuroscience 9, 370 (2017).

15. G. Chen et al., Abnormal cerebellum-DMN regions connectivity in unmedicated bipolar II disorder. Journal of affective disorders 243, 441-447 (2019).

16. L. Douw et al., Dissociated multimodal hubs and seizures in temporal lobe epilepsy. Annals of clinical and translational neurology 2, 338-352 (2015).

17. H. A. Eyre et al., Altered resting-state functional connectivity in late-life depression: A cross-sectional study. Journal of affective disorders 189, 126-133 (2016).

18. V. Balaev, I. Orlov, A. Petrushevsky, O. Martynova, Functional connectivity between salience, default mode and frontoparietal networks in post-stroke depression. Journal of affective disorders 227, 554-562 (2018).

19. V. Bonnelle et al., Salience network integrity predicts default mode network function after traumatic brain injury. Proceedings of the National Academy of Sciences of the United States of America 109, 4690-4695 (2012).

20. Y. T. Chang et al., Salience Network and Depressive Severities in Parkinson's Disease with Mild Cognitive Impairment: A Structural Covariance Network Analysis. Frontiers in aging neuroscience 9 , 417 (2017).

21. M. P. Catalino et al., Mapping cognitive and emotional networks in neurosurgical patients using resting-state functional magnetic resonance imaging. Neurosurgical focus 48, E9 (2020).

22. M. Corbetta, G. L. Shulman, Spatial neglect and attention networks. Annual review of neuroscience 34, 569-599 (2011).

23. N. B. Dadario, B. Brahimaj, J. Yeung, M. E. Sughrue, Reducing the Cognitive Footprint of Brain Tumor Surgery. Frontiers in Neurology 12, 1342 (2021). 
24. J. T. Yeung et al., Using Quicktome for intracerebral surgery: early retrospective study and proof of concept. World neurosurgery 10.1016/j.wneu.2021.07.127 (2021).

25. E. Garyfallidis et al., Dipy, a library for the analysis of diffusion MRI data. Frontiers in Neuroinformatics 8 (2014).

26. M. F. Glasser et al., A multi-modal parcellation of human cerebral cortex. Nature 536, 171-178 (2016).

27. H. Ren et al., Application of Structural and Functional Connectome Mismatch for Classification and Individualized Therapy in Alzheimer Disease. Frontiers in public health 8, 584430 (2020).

28. R. G. Briggs et al., A Connectomic Atlas of the Human Cerebrum-Chapter 18: The Connectional Anatomy of Human Brain Networks. Operative neurosurgery (Hagerstown, Md.) 15, S470-s480 (2018).

29. P. G. Allan et al., Parcellation-based tractographic modeling of the dorsal attention network. Brain Behav 9, e01365 (2019).

30. P. G. Allan et al., Parcellation-based tractographic modeling of the ventral attention network. $J$ Neurol Sci 408, 116548 (2020).

31. J. J. Kuiper et al., A parcellation-based model of the auditory network. Hear Res 396, 108078 (2020).

32. J. R. Sheets et al., Parcellation-based modeling of the dorsal premotor area. Journal of the neurological sciences 415, 116907 (2020).

33. J. R. Sheets et al., A Cortical Parcellation Based Analysis of Ventral Premotor Area Connectivity. Neurological research 43, 595-607 (2021).

\section{Figures}

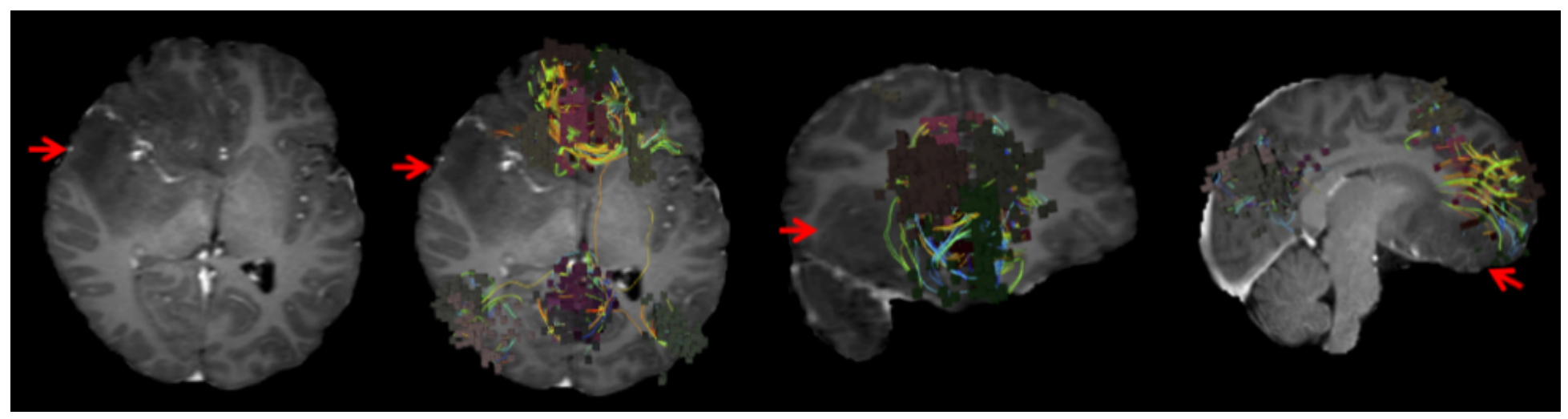

\section{Figure 1}

Default mode network (DMN) invaded by tumor 


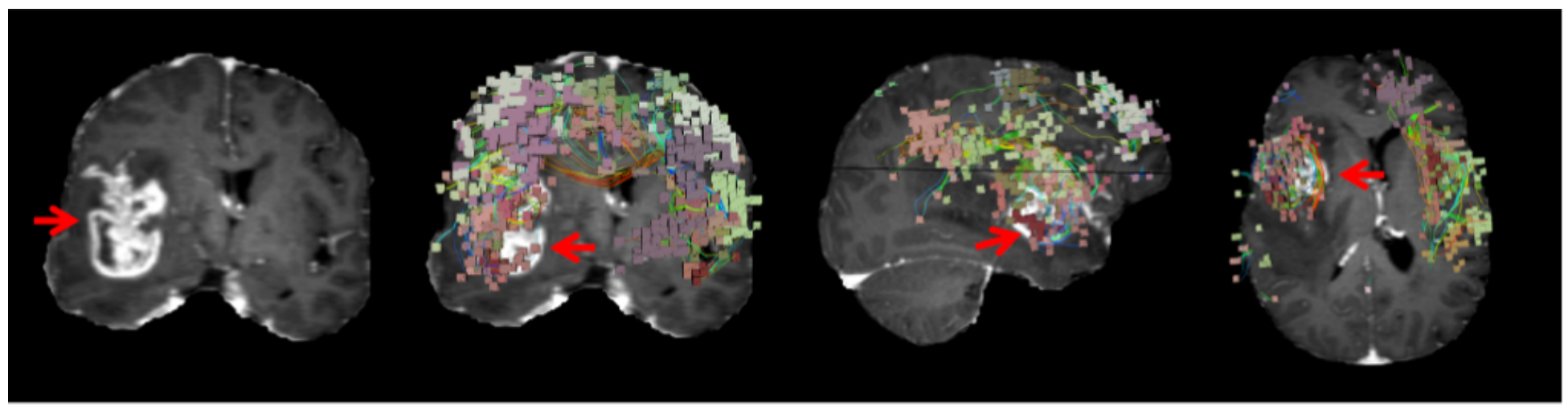

Figure 2

Salience network (SN) invaded by tumor

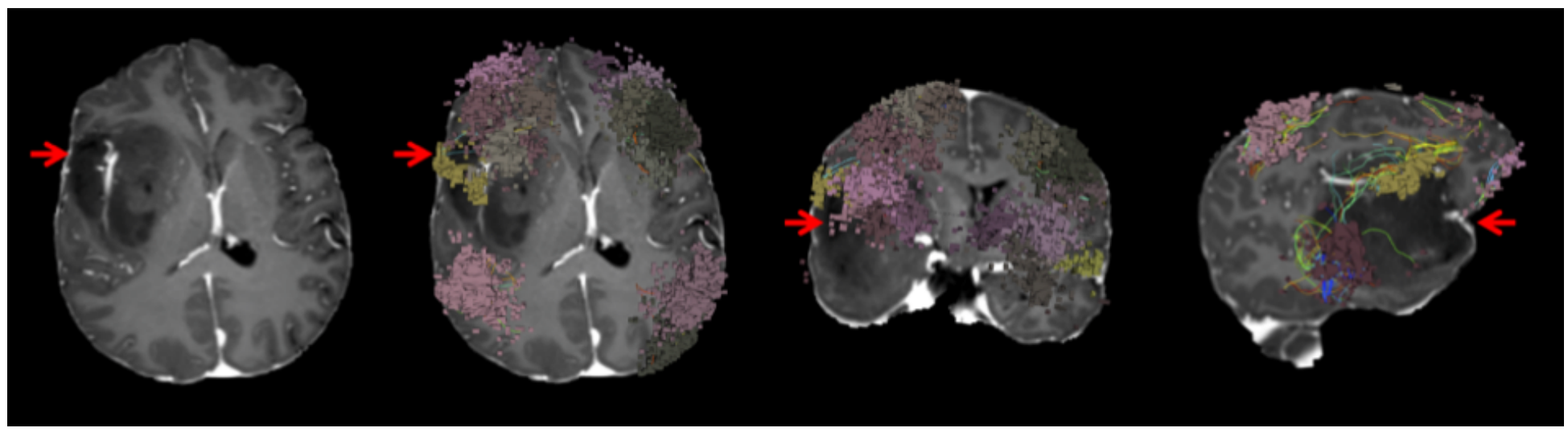

Figure 3

Central executive network (CEN) invaded by tumor

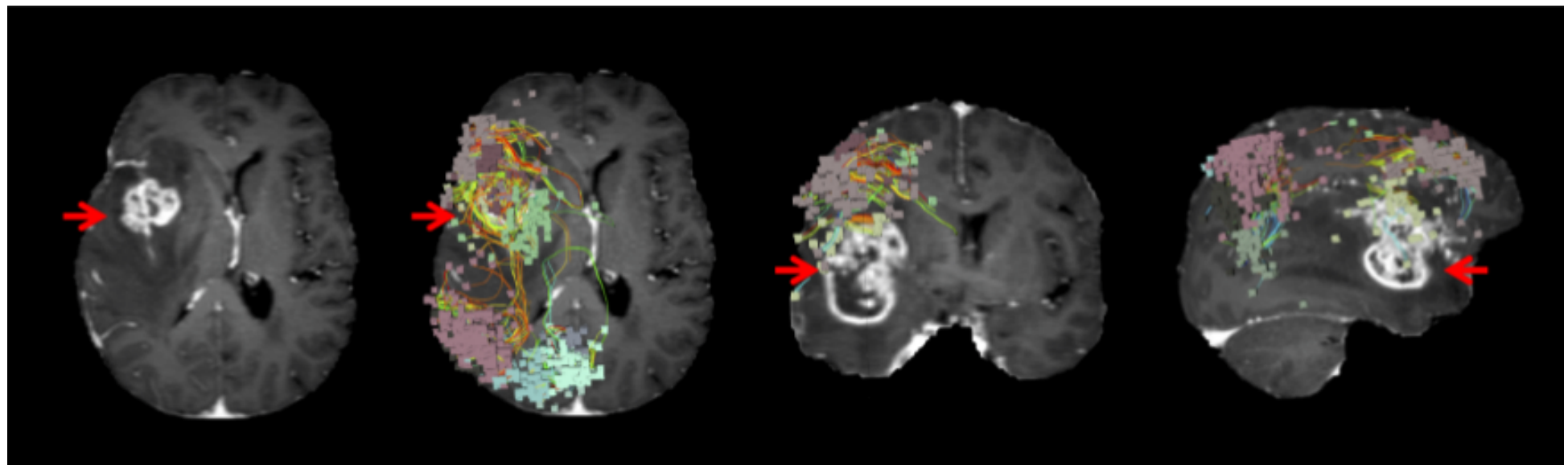

Figure 4

Ventral attention network (VAN) network invaded by tumor 


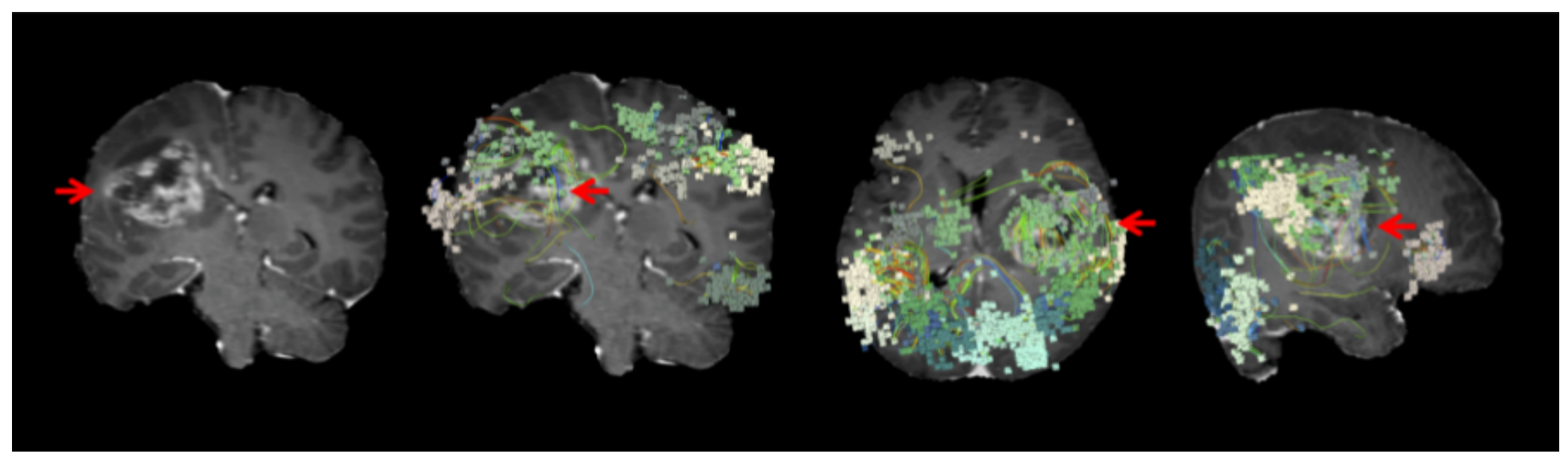

Figure 5

Dorsal attention network (DAN) network invaded by tumor 